\title{
Valuation of American Call Option Considering Uncertain Volatility
}

\author{
I. Hlaváček ${ }^{1, *}$ \\ ${ }^{1}$ Institute of Mathematics, Academy of Sciences of the Czech Republic, \\ Žitná 25, Prague 1, CZ 115 67, Czech Republic
}

Received 20 July 2009; Accepted (in revised version) 30 September 2009

Available online 5 March 2010

\begin{abstract}
The parabolic variational inequality for simulating the valuation of American option is used to analyze a continuous dependence of the solution with respect to the uncertain volatility parameter. Three kinds of the continuity are proved, enabling us to employ the maximum range method for the uncertain parameter, under the condition that the criterion-functional has the corresponding property.
\end{abstract}

AMS subject classifications: 91B28, 49J40, 49N30.

Key words: American options, parabolic variational inequality, uncertain parameter.

\section{Introduction}

The problem of pricing American options is important both in theory and in practice. It has been shown by the Nobel Prize laureates Merton [7] and Black and Scholes [4] that the valuation of American call option can be simulated by a free boundary problem for a degenerate parabolic equation. A weak solution of the problem has been defined by Badea and Wang [2]. They proved the existence and uniqueness of the weak solution and some regularity results by a detailed analysis based on the use of maximum principles.

Efficient numerical methods for the solution of the problem using finite elements in "space" and backward differences in "time" have been proposed by Allegretto et al. [1] and Lin et al. [8]. These authors started from an equivalent variational inequality, which can be derived by a suitable change of all variables and which avoids the degeneracy.

The aim of the present paper is to complete the results of Badea and Wang $[2,3]$ by an analysis of a continuous dependence of the weak solution with respect to the volatility. The latter parameter appears to be the only parameter, which is not observable directly in the market. On the basis of a variational inequality for the weak

${ }^{*}$ Corresponding author.

Email: hlavacek@math.cas.cz (I. Hlaváček) 
solution [3], we prove three kinds of the continuous dependence, provided the volatility belongs to a given compact interval of positive numbers. Then one can employ the maximum range method [5,6], if a criterion-functional is prescribed, which has properties, corresponding to the three kinds of the continuity, mentioned above.

\section{Formulation by a parabolic variational inequality}

The original mathematical model of American call option proposed by Merton [7] is represented by a free boundary problem for a parabolic equation

$$
w_{t}-\frac{1}{2} \sigma^{2} x^{2} w_{x x}-(r-d) x w_{x}+r w=0, \quad \text { in } D,
$$

with the initial condition

$$
w(x, 0)=0
$$

and the boundary conditions

$$
w(0, t)=0, \quad w(s(t), t)=(x-Z)^{+}, \quad w_{x}(s(t), t)=1,
$$

for all $t \in(0, T]$. Here $w(x, t)$ denotes the value of the American call option, $T$ is the maturity date (the time at which the American call option expires), $r$ is the interest rate, $d$ the dividend rate, $\sigma$ the volatility, $Z$ the exercise price, $x$ denotes the stock price, $t \equiv T-t_{r}$, where $t_{r}$ is the real time,

$$
D=\{(x, t): 0<x<s(t), \quad t \in(0, T]\} .
$$

The free boundary $x=s(t)$ denotes the optimal exercise curve,

$$
w_{x} \equiv \frac{\partial w}{\partial x}, \quad w_{t} \equiv \frac{\partial w}{\partial t}, \quad \text { and } \quad(u)^{+}=\max \{u, 0\}
$$

We assume that $r, d, Z, \sigma$ are positive real constants.

If we define a new function $u(x, t)$ by

$$
u=w-(x-Z)^{+},
$$

we can extend the function $u$ outside the domain $D$ by zero. In this way a weak solution has been defined by Badea and Wang in [2], where an upper bound

$$
S_{0}(\sigma)=\frac{Z \lambda(\sigma)}{(\lambda(\sigma)-1)}
$$

with

$$
\lambda(\sigma)=\sigma^{-2}\left\{\frac{\sigma^{2}}{2}-r+d+\left[\left(\frac{\sigma^{2}}{2}-r+d\right)^{2}+2 r \sigma^{2}\right]^{\frac{1}{2}}\right\}
$$


was found for $s(t)$, so that

$$
\sup _{t \in(0, T]} s(t) \leq S_{0}
$$

Remark 2.1. Since $d>0$ and $\sigma^{2}>0, \lambda(\sigma)>1$ follows. One can choose any constant $S \geq S_{0}(\sigma)$ and define $\Omega=(0, S), \quad I=[0, T]$, the space

$$
W(I)=\left\{v \in L^{2}\left(I, H_{0}^{1}(\Omega)\right): x^{-1} v_{t} \in L^{2}\left(I, L^{2}(\Omega)\right)\right\},
$$

the bilinear form

$$
a(\sigma ; u, v)=\frac{\sigma^{2}}{2\left(u_{x}, v_{x}\right)}+(d-r)\left(u_{x}, x^{-1} v\right)+r\left(x^{-1} u, x^{-1} v\right),
$$

and the function

$$
q(x)=\left(x^{-1} d-x^{-2} r Z\right) H(x-Z),
$$

where $(f, g)$ denotes the inner product $\int_{\Omega} f g d x$ and $H(\cdot)$ is the Heaviside function.

Definition 2.1. We say that $u$ is a weak solution if $u \in W(I)$ is such that $u(x, 0)=0$ and

$$
\left(x^{-2} u_{t}, v\right)+a(\sigma ; u, v)+(q H(u), v)=\frac{1}{2} \sigma^{2} v(Z),
$$

holds for all $v \in H_{0}^{1}(\Omega)$ and almost all $t \in I$.

Badea and Wang proved in [2] that there exists a unique weak solution,

$$
\begin{array}{ll}
x^{-1} u \in L^{\infty}\left(I, L^{2}(\Omega)\right), & u_{x} \in L^{\infty}\left(I, L^{2}(\Omega)\right), \\
\sup _{t \in(0, T]}\{s(t)\} \leq S_{0}, & \inf _{t \in I}\{s(t)\} \geq Z \max \left\{\frac{r}{d}, 1\right\}, \\
u_{t} \geq 0, \quad \text { in } \Omega \times I, & u>0, \quad \text { in } D, \\
u=0, \quad \text { in }(\Omega \times I) \backslash D . &
\end{array}
$$

Moreover, the values of $u(x, t)$ do not depend on the value of $S$. It is easy to deduce that the weak solution satisfies the following parabolic variational inequality (see [3]):

$$
\begin{aligned}
& \left(x^{-2} u_{t}, v-u\right)+a(\sigma ; u, v-u) \\
\geq & \frac{\sigma^{2}(v(Z)-u(Z))}{2}-\int_{Z}^{S} q(v-u) d x
\end{aligned}
$$

for all $v \in K$ and

$$
\text { almost all } t \in I, \quad u \in L^{2}(I, K), \quad x^{-1} u_{t} \in L^{2}\left(I, L^{2}(\Omega)\right),
$$

and $u(x, 0)=0$, where

$$
K=\left\{v \in H_{0}^{1}(\Omega): v \geq 0, \quad \text { in } \Omega\right\} .
$$

In what follows we will start the analysis on the basis of problem (2.4). 


\section{Continuous dependence of solution with respect to volatility}

We will consider that the volatility $\sigma$ is uncertain and belongs to a given interval

$$
\mathcal{U}_{a d}=\left[\sigma_{\min }, \sigma_{\max }\right], \quad 0<\sigma_{\min }<\sigma_{\max }<\infty .
$$

A question arises about the dependence of the solution $u \equiv u(\sigma)$ of problem (2.4) on the parameter $\sigma \in \mathcal{U}_{a d}$. Let us choose

$$
S \geq \max _{\sigma \in \mathcal{U}_{a d}} S_{0}(\sigma)
$$

Then we will prove the following main result.

Theorem 3.1. Let $\sigma_{n} \in \mathcal{U}_{a d}, \sigma_{n} \rightarrow \sigma$, as $n \rightarrow \infty$. Then

$$
\begin{array}{ll}
u\left(\sigma_{n}\right) \rightarrow u(\sigma), & \text { in } L^{2}\left(I ; H_{0}^{1}(\Omega)\right), \\
x^{-1} u\left(\sigma_{n}\right)(t) \rightarrow x^{-1} u(\sigma)(t), & \text { for a.a. } t \in I, \\
x^{-1} u\left(\sigma_{n}\right) \rightarrow x^{-1} u(\sigma) \quad(\text { weak star }), & \text { in } L^{\infty}\left(I ; L^{2}(\Omega)\right) .
\end{array}
$$

For the proof we shall need the following lemmas and a proposition.

Lemma 3.1. There exist positive constants $C_{0}, C_{1}$ such that

$$
\begin{aligned}
& a(\sigma ; v, v)=\frac{\sigma^{2}}{2\left\|v_{x}\right\|_{0}^{2}}+\frac{r+d}{2\left\|x^{-1} v\right\|_{0}^{2}} \geq C_{0}\|v\|_{1}^{2}, \\
& |a(\sigma ; u, v)| \leq C_{1}\|u\|_{1}\|v\|_{1},
\end{aligned}
$$

for any $u, v \in H_{0}^{1}(\Omega)$ and any $\sigma \in \mathcal{U}_{\text {ad }}$.

Henceforth $\|v\|_{0}$ denotes the norm in $L^{2}(\Omega)$ and $\|v\|_{1}$ the standard norm in the Sobolev space $H^{1}(\Omega)$.

Proof is a consequence of the inequalities

$$
S^{-1}\|v\|_{0} \leq\left\|x^{-1} v\right\|_{0} \leq 2\left\|v_{x}\right\|_{0}, \quad \forall v \in H_{0}^{1}(\Omega) .
$$

Proposition 3.2. There exists a constant $C_{3}$ such that

$$
\left\|x^{-1} u_{t}(\sigma)\right\|_{L^{2}\left(I ; L^{2}(\Omega)\right)} \leq C_{3}, \quad \forall \sigma \in \mathcal{U}_{a d}
$$

Proof. By an analysis of the proof of Lemmas 3.4 and 3.5 in [2]-II, we infer that

$$
\left\|x^{-1} \partial_{t} u_{k}(\sigma)\right\|_{L^{2}\left(I ; L^{2}(\Omega)\right)} \leq C\left(\sigma_{\max }\right), \quad \forall \sigma \in \mathcal{U}_{a d}
$$


where $u_{k}(\sigma)$ is the Rothe piecewise linear approximation of $u(\sigma)$ in the interval $I$. Then a function $\omega$ with

$$
x^{-1} \partial_{t} \omega \in L^{2}\left(I ; L^{2}(\Omega)\right),
$$

can be found as a weak limit of a subsequence of $\left\{x^{-1} \partial_{t} u_{k}(\sigma)\right\}$ such that the norm of $x^{-1} \partial_{t} \omega$ in $L^{2}\left(I ; L^{2}(\Omega)\right)$ is bounded by $C\left(\sigma_{\max }\right)$. In proving Theorems 4.1 and 2.1 of [2]-II, it was verified that $\omega \equiv u(\sigma)$.

Lemma 3.2. The set $L^{2}\left(I_{t} ; K\right)$ is weakly closed in

$$
L^{2}\left(I_{t} ; H_{0}^{1}(\Omega)\right), \quad \text { for all } t \in I,
$$

where $I_{t}=(0, T]$.

Proof. It is readily seen that $L^{2}\left(I_{t} ; K\right)$ is convex, since $K$ is convex. Let $u_{n} \in$ $L^{2}\left(I_{t} ; K\right), u_{n} \rightarrow u$ in $L^{2}\left(I_{t} ; H_{0}^{1}(\Omega)\right)$, as $n \rightarrow \infty$. Then from

$$
\int_{0}^{t}\left\|u_{n}(\tau)-u(\tau)\right\|_{1}^{2} d \tau \rightarrow 0, \quad \forall t \in I,
$$

we infer that $u_{n}(\tau) \rightarrow u(\tau)$ in $H_{0}^{1}(\Omega)$ for a.a. $\tau \in I_{t}$, so that $u(\tau) \in K$ for a.a. $\tau \in I_{t}$ and $u \in L^{2}\left(I_{t} ; K\right)$. Since $L^{2}\left(I_{t} ; K\right)$ is convex and closed in $L^{2}\left(I_{t} ; H_{0}^{1}(\Omega)\right)$, it is weakly closed.

Proof of Theorem 3.1. For brevity, let us denote $u_{n}=u\left(\sigma_{n}\right)$ and

$$
L\left(\sigma_{n} ; w\right)=\frac{\sigma^{2} w(Z)}{2}-\int_{Z}^{S} q w d x
$$

From (2.4), we infer that

$$
\left(x^{-2} u_{n t}, v-u_{n}\right)+a\left(\sigma_{n} ; u_{n}, v-u_{n}\right) \geq L\left(\sigma_{n} ; v-u_{n}\right),
$$

holds for any $v \in K$ and a.a. $t \in I$.

Let us insert $v=0$ to obtain

$$
\left(x^{-1} \partial_{t} u_{n}, x^{-1} u_{n}\right)+a\left(\sigma ; u_{n}, u_{n}\right) \leq L\left(\sigma_{n} ; u_{n}\right) .
$$

By using Lemma 3.1, the embedding $H_{0}^{1}(\Omega) \hookrightarrow C(\bar{\Omega})$ and the inequality

$$
|f g| \leq \frac{\epsilon}{2 f^{2}}+(2 \epsilon)^{-1} g^{2}
$$

with $\epsilon>0$, we arrive at

$$
\frac{d}{d t}\left\|x^{-1} u_{n}(t)\right\|_{0}^{2}+2 C_{0}\left\|u_{n}\right\|_{1}^{2} \leq C_{0}\left\|u_{n}\right\|_{1}^{2}+\bar{C}
$$


Integrating over the interval $I_{t}$, we obtain

$$
\left\|x^{-1} u_{n}(t)\right\|_{0}^{2}+C_{0} \int_{0}^{t}\left\|u_{n}(\tau)\right\|_{1}^{2} d \tau \leq \bar{C} T, \quad \forall t \in I, \quad n \geq 1,
$$

Then there exists a subsequence $\left\{u_{m}\right\} \subset\left\{u_{n}\right\}$ and $u^{*}$ such that

$$
x^{-1} u^{*}(t) \in L^{2}(\Omega), \quad u^{*} \in L^{2}\left(I ; H_{0}^{1}(\Omega)\right),
$$

and

$$
\begin{array}{ll}
x^{-1} u_{m}(t) \rightarrow x^{-1} u^{*}(t) \quad \text { (weakly), } & \text { in } L^{2}(\Omega), \forall t \in I, \\
x^{-1} u_{m} \rightarrow x^{-1} u^{*} \text { (weak star), } & \text { in } L^{\infty}\left(I ; L^{2}(\Omega)\right), \\
u_{m} \rightarrow u^{*} \text { (weakly), } & \text { in } L^{2}\left(I ; H_{0}^{1}(\Omega)\right) .
\end{array}
$$

Let us verify that $u^{*}=u(\sigma)$. To this end we choose an arbitrary $v \in L^{2}\left(I_{t} ; K\right)$. We have

$$
\left(x^{-2} \partial_{t} u_{m}, u_{m}-v\right)+a\left(\sigma_{m} ; u_{m}, u_{m}-v\right) \leq L\left(\sigma_{m} ; u_{m}-v\right),
$$

for almost all $t \in I$. From this inequality, we obtain

$$
\begin{aligned}
& \frac{d}{2 d t}\left\|x^{-1} u_{m}\right\|_{0}^{2}+a\left(\sigma_{m} ; u_{m}, u_{m}\right) \\
\leq & \left(x^{-1} \partial_{t} u_{m}, v\right)+a\left(\sigma_{m} ; u_{m}, v\right)+L\left(\sigma_{m} ; u_{m}-v\right),
\end{aligned}
$$

so that

$$
\begin{gathered}
\frac{1}{2}\left\|x^{-1} u_{m}(t)\right\|_{0}^{2}+\int_{0}^{t} a\left(\sigma_{m} ; u_{m}(\tau), u_{m}(\tau)\right) d \tau \\
\leq \int_{0}^{t}\left[\left(x^{-1} \partial_{t} u_{m}(\tau), v(\tau)\right)+a\left(\sigma_{m} ; u_{m}(\tau), v(\tau)\right)\right. \\
\left.+L\left(\sigma_{m} ; u_{m}(\tau)-v(\tau)\right)\right] d \tau,
\end{gathered}
$$

holds for all $t \in I$.

Let us pass to the $\lim \inf$ with $m \rightarrow \infty$. By virtue of (3.4), we arrive at

$$
\liminf \left\|x^{-1} u_{m}(t)\right\|_{0}^{2} \geq\left\|x^{-1} u^{*}(t)\right\|_{0}^{2} .
$$

The functional

$$
\psi(w)=\int_{0}^{t} a(\sigma ; w(\tau), w(\tau)) d \tau
$$

is convex and continuous in $L^{2}\left(I ; H_{0}^{1}(\Omega)\right)$ due to Lemma 3.1. Hence, it is weakly lower semicontinuous, so that (3.6) yields

$$
\liminf \int_{0}^{t} a\left(\sigma ; u_{m}(\tau), u_{m}(\tau)\right) d \tau \geq \int_{0}^{t} a\left(\sigma ; u^{*}(\tau), u^{*}(\tau)\right) d \tau
$$


Moreover, we observe that

$$
\begin{aligned}
& \quad\left|\int_{0}^{t} a\left(\sigma_{m} ; u_{m}, u_{m}\right) d \tau-\int_{0}^{t} a\left(\sigma ; u_{m}, u_{m}\right) d \tau\right| \\
& \leq \frac{1}{2} \int_{0}^{t}\left|\sigma_{m}^{2}-\sigma^{2}\right|\left\|u_{m}(\tau)\right\|_{1}^{2} d \tau \rightarrow 0,
\end{aligned}
$$

follows from (3.3).

By using Proposition 3.1, we infer that a subsequence of $\left\{u_{m}\right\}$ exists (and we shall denote it by the same symbol), such that

$$
x^{-1} \partial_{t} u_{m} \rightarrow x^{-1} \partial_{t} u^{*}(\text { weakly }), \quad \text { in } L^{2}\left(I ; L^{2}(\Omega)\right), \quad \text { as } m \rightarrow \infty .
$$

Then

$$
\int_{0}^{t}\left(x^{-1} \partial_{t} u_{m}, x^{-1} v\right) d \tau \rightarrow \int_{0}^{t}\left(x^{-1} \partial_{t} u^{*}, x^{-1} v\right) d \tau
$$

Since

$$
x^{-1} v(\tau) \in L^{2}(\Omega), \quad \text { if } \quad v(\tau) \in H_{0}^{1}(\Omega),
$$

so that the integral represents a linear continuous functional in $L^{2}\left(I, L^{2}(\Omega)\right)$. By virtue of (3.6)

$$
\int_{0}^{t} a\left(\sigma ; u_{m}, v\right) d \tau \rightarrow \int_{0}^{t} a\left(\sigma ; u^{*}, v\right) d \tau
$$

and using an analogue of (3.11), we arrive at

$$
\int_{0}^{t} a\left(\sigma ; u_{m}, v\right) d \tau \rightarrow \int_{0}^{t} a\left(\sigma ; u^{*}, v\right) d \tau .
$$

Next, we have

$$
\begin{aligned}
& \quad\left|\int_{0}^{t} L\left(\sigma_{m} ; u_{m}-v\right) d \tau-\int_{0}^{t} L\left(\sigma ; u_{m}-v\right) d \tau\right| \\
& \leq C\left|\sigma_{m}^{2}-\sigma^{2}\right| \int_{0}^{t}\left\|u_{m}-v\right\|_{1} d \tau \rightarrow 0,
\end{aligned}
$$

and

$$
\int_{0}^{t} L\left(\sigma ; u_{m}-v\right) d \tau \rightarrow \int_{0}^{t} L\left(\sigma ; u^{*}-v\right) d \tau,
$$

by virtue of (3.6). As a result,

$$
\int_{0}^{t} L\left(\sigma_{m} ; u_{m}-v\right) d \tau \rightarrow \int_{0}^{t} L\left(\sigma ; u^{*}-v\right) d \tau .
$$


From (3.7)-(3.14), we obtain

$$
\begin{aligned}
& \frac{1}{2}\left\|x^{-1} u^{*}(t)\right\|_{0}^{2}+\int_{0}^{t} a\left(\sigma ; u^{*}, u^{*}\right) d \tau \\
\leq & \int_{0}^{t}\left[\left(x^{-1} \partial_{t} u^{*}, x^{-1} v\right)+a\left(\sigma ; u^{*}, v\right)+L\left(\sigma ; u^{*}-v\right)\right] d \tau .
\end{aligned}
$$

This inequality can be rewritten as

$$
\int_{0}^{t}\left[\left(x^{-1} \partial_{t} u^{*}, x^{-1} v-x^{-1} u^{*}\right)+a\left(\sigma ; u^{*}, v-u^{*}\right)-L\left(\sigma ; v-u^{*}\right)\right] d \tau \geq 0,
$$

By Lemma 3.2 and (3.6), we infer that $u^{*} \in L^{2}\left(I_{t}, K\right)$.

Let us set

$$
v(\tau)=u^{*}(\tau)+w(x) \chi_{\epsilon}(\tau),
$$

where $w \in K$ is arbitrary and $\chi_{\epsilon}$ is the characteristic function of the interval

$$
\left[t_{0}-\epsilon, t_{0}+\epsilon\right], \quad t_{0}<T .
$$

Then $v \in L^{2}\left(I_{t}, K\right)$. Since the Lebesgue Theorem implies that

$$
(2 \epsilon)^{-1} \int_{t_{0}-\epsilon}^{t_{0}+\epsilon} g(\tau) d \tau \rightarrow g\left(t_{0}\right), \quad \text { as } \quad \epsilon \rightarrow 0,
$$

holds for any measurable function $g$ and almost all $t_{0} \in I$, from (3.15) we infer that

$$
\left[\left(x^{-1} \partial_{t} u^{*}, x^{-1} w\right)+a\left(\sigma ; u^{*}, w\right)-L(\sigma ; w)\right]_{t=t_{0}} \geq 0 .
$$

Next, let us take $v=0$ and $v=2 u^{*}$ in (3.15). Then

$$
\int_{0}^{t}\left[\left(x^{-1} \partial_{t} u^{*}, x^{-1} u^{*}\right)+a\left(\sigma ; u^{*}, u^{*}\right)-L\left(\sigma ; u^{*}\right)\right] d \tau=0, \quad \forall t \in I,
$$

and

$$
\left[\left(x^{-1} \partial_{t} u^{*}, x^{-1} u^{*}\right)+a\left(\sigma ; u^{*}, u^{*}\right)-L\left(\sigma ; u^{*}\right)\right]_{t=t_{0}},
$$

follows by differentiation. From (3.16) and (3.18), we obtain

$$
\left(x^{-2} \partial_{t} u^{*}, w-u^{*}\right)+a\left(\sigma ; u^{*}, w-u^{*}\right)-L\left(\sigma ; w-u^{*}\right) \geq 0,
$$

for all $w \in K$ and almost all $t \in I$, so that $u^{*}=u(\sigma)$.

Since the weak solution is unique (see [2]-I), the whole original sequence $\left\{u_{n}\right\}$ tends to $u(\sigma)$ weakly in $L^{2}\left(I, H_{0}^{1}(\Omega)\right), x^{-1} u_{n}(t)$ tends weakly to $x^{-1} u(\sigma)(t)$ in $L^{2}(\Omega)$ for all $t \in I$ and $x^{-1} u_{n}$ weakly star to $x^{-1} u(\sigma)$ in $L^{\infty}\left(I, L^{2}(\Omega)\right)$.

To prove the strong convergence, we introduce the following bilinear form in $L^{2}\left(I_{t}, H_{0}^{1}(\Omega)\right)$

$$
\langle u, v\rangle=\frac{1}{2}\left(x^{-1} u(t), x^{-1} v(t)\right)+\int_{0}^{t} a(\sigma ; u, v) d \tau,
$$

and let $\|u\|_{a}^{2}=\langle u, u\rangle$. 
Lemma 3.3. Let us denote $u=u(\sigma), u_{n}=u\left(\sigma_{n}\right)$. Then

$$
\left\|u_{n}\right\|_{a} \rightarrow\|u\|_{a}, \quad \text { as } n \rightarrow \infty \text {. }
$$

Proof. Since $\left\{u_{n}\right\}$ is bounded in $L^{2}\left(I, H_{0}^{1}(\Omega)\right)$ by virtue of (3.3),

$$
\int_{0}^{t}\left(a\left(\sigma_{n} ; u_{n}, u_{n}\right)-a\left(\sigma ; u_{n}, u_{n}\right)\right) d \tau \leq \frac{1}{2}\left|\sigma_{n}^{2}-\sigma^{2}\right| \int_{0}^{t}\left\|u_{n}\right\|_{1}^{2} d \tau \rightarrow 0
$$

and

$$
\left|\int_{0}^{t} L\left(\sigma_{n} ; u_{n}\right) d \tau-\int_{0}^{t} L\left(\sigma ; u_{n}\right) d \tau\right| \leq C\left|\sigma_{n}^{2}-\sigma^{2}\right| \int_{0}^{t}\left\|u_{n}\right\|_{1} d \tau \rightarrow 0 .
$$

In the variational inequality for $u_{n}$, we insert $v=0$ and $v=2 u_{n}$ to obtain

$$
\begin{aligned}
\mathcal{B}_{n} & \equiv \frac{\left\|x^{-1} u_{n}(t)\right\|^{2}}{2}+\int_{0}^{t} a\left(\sigma_{n} ; u_{n}, u_{n}\right) d \tau \\
& =\int_{0}^{t} L\left(\sigma_{n} ; u_{n}\right) d \tau .
\end{aligned}
$$

Then from (3.19)-(3.21) and the weak convergence of $\left\{u_{n}\right\}$ it follows that

$$
\begin{aligned}
\left\|u_{n}\right\|_{a}^{2} & =\mathcal{B}_{n}+\int_{0}^{t}\left(a\left(\sigma ; u_{n}, u_{n}\right)-a\left(\sigma_{n} ; u_{n}, u_{n}\right)\right) d \tau \\
& =\int_{0}^{t} L\left(\sigma ; u_{n}\right) d \tau+\int_{0}^{t}\left(L\left(\sigma_{n} ; u_{n}\right)-L\left(\sigma ; u_{n}\right)\right) d \tau \\
& \rightarrow \int_{0}^{t} L(\sigma ; u) d \tau .
\end{aligned}
$$

On the other hand, (3.17) yields that

$$
\|u\|_{a}^{2}=\int_{0}^{t} L(\sigma ; u) d \tau
$$

so that

$$
\left\|u_{n}\right\|_{a}^{2} \rightarrow\|u\|_{a^{\prime}}^{2} \quad \text { as } \quad n \rightarrow \infty
$$

Then the lemma is proved.

Let us observe that

$$
\left\langle u_{n}, v\right\rangle=\frac{1}{2}\left(x^{-1} u_{n}(t), x^{-1} v\right)+\int_{0}^{t} a\left(\sigma ; u_{n}, v\right) d \tau \rightarrow\langle u, v\rangle,
$$

and $\left\langle v, u_{n}\right\rangle \rightarrow\langle v, u\rangle$ holds for any $v \in L^{2}\left(I_{t}, H_{0}^{1}(\Omega)\right)$ by virtue of the weak convergences proved above. Then

$$
\begin{aligned}
\left\|u_{n}-u\right\|_{a}^{2} & =\left\langle u_{n}-u, u_{n}-u\right\rangle \\
& =\left\|u_{n}\right\|_{a}^{2}+\|u\|_{a}^{2}-\left\langle u, u_{n}\right\rangle-\left\langle u_{n}, u\right\rangle \rightarrow 0
\end{aligned}
$$


follows from Lemma (3.3).

It is readily seen that

$$
C_{0} \int_{0}^{t}\|v\|_{1}^{2} d \tau \leq\|v\|_{a}^{2} \quad \text { and } \quad \frac{1}{2}\left\|x^{-1} v(t)\right\|_{0}^{2} \leq\|v\|_{a}^{2},
$$

hold for any $v \in L^{2}\left(I ; H_{0}^{1}(\Omega)\right)$. Then the strong convergence follows from (3.22).

\section{Maximum range problem}

Assume that a criterion-functional

$$
\Phi(\sigma ; v): \mathcal{U}_{a d} \times\left(L_{x^{-1}}^{2}(\Omega) \times L^{2}\left(I, H_{0}^{1}(\Omega)\right)\right) \mapsto \mathbf{R},
$$

is given, such that if

$$
\begin{array}{ll}
\sigma_{n} \in \mathcal{U}_{a d}, \quad \sigma_{n} \rightarrow \sigma, \quad \text { as } \quad n \rightarrow \infty, & \\
\text { (i) } v_{n} \rightarrow v, & \text { in } L^{2}\left(I, H_{0}^{1}(\Omega)\right), \\
\text { (ii) } x^{-1} v_{n}(t) \rightarrow x^{-1} v(t), & \text { in } L^{2}(\Omega), \forall t \in I, \\
\text { (iii) } x^{-1} v_{n} \rightarrow x^{-1} v \text { (weakstar), } & \text { in } L^{\infty}\left(I, L^{2}(\Omega)\right),
\end{array}
$$

then

$$
\Phi\left(\sigma_{n} ; v_{n}\right) \rightarrow \Phi(\sigma ; v), \quad \text { as } n \rightarrow \infty
$$

Theorem 4.1. Let the criterion-functional $\Phi$ satisfy conditions (4.1)-(4.2). Then there exists at least one solution of the maximization problem

$$
\bar{\sigma}=\arg \max _{\sigma \in \mathcal{U}_{a d}} \Phi(\sigma ; u(\sigma)),
$$

and at least one solution of the minimization problem

$$
\underline{\sigma}=\arg \min _{\sigma \in \mathcal{U}_{a d}} \Phi(\sigma ; u(\sigma)) .
$$
i.e.,

Proof. $1^{0}$. Let $\left\{\sigma_{n}\right\}$ be a maximizing sequence of the functional $J(\sigma) \equiv \Phi(\sigma ; u(\sigma))$,

$$
J\left(\sigma_{n}\right) \rightarrow \sup _{\sigma \in \mathcal{U}_{a d}} J(\sigma), \quad \text { as } \quad n \rightarrow \infty .
$$

Since $\mathcal{U}_{a d}$ is compact, there exists a subsequence $\left\{\sigma_{m}\right\}$ and $\sigma^{*} \in \mathcal{U}_{a d}$ such that

$$
\sigma_{m} \rightarrow \sigma^{*}, \quad \text { as } \quad m \rightarrow \infty .
$$

By Theorem 3.1, the sequence $\left\{u\left(\sigma_{m}\right)\right\}$ and $u\left(\sigma^{*}\right)$ satisfy conditions (i)-(iii) of (4.1). By using assumption (4.1)-(4.2), we infer that

$$
\begin{aligned}
J\left(\sigma_{m}\right) & =\Phi\left(\sigma_{m} ; u\left(\sigma_{m}\right)\right) \rightarrow \Phi\left(\sigma^{*} ; u\left(\sigma^{*}\right)\right) \\
& =J\left(\sigma^{*}\right), \quad \text { as } \quad m \rightarrow \infty .
\end{aligned}
$$


From (4.5), we obtain

$$
J\left(\sigma^{*}\right)=\sup _{\sigma \in \mathcal{U}_{a d}} J(\sigma),
$$

so that $\sigma^{*}$ solves problem (4.3), i.e., $\sigma^{*}=\bar{\sigma}$.

$2^{0}$. Let $\left\{\sigma_{n}\right\}$ be a minimizing sequence of $J(\sigma)$, i.e.,

$$
J\left(\sigma_{n}\right) \rightarrow \inf _{\sigma \in \mathcal{U}_{a d}} J(\sigma), \quad \text { as } \quad n \rightarrow \infty
$$

There exist $\left\{\sigma_{m}\right\} \subset\left\{\sigma_{n}\right\}$ and $\sigma_{*}$ such that

$$
\sigma_{m} \rightarrow \sigma_{*}, \quad \text { as } \quad m \rightarrow \infty
$$

Using Theorem 3.1, we obtain $J\left(\sigma_{m}\right) \rightarrow J\left(\sigma_{*}\right)$ and by comparing the limit with (4.6), we arrive at

$$
J\left(\sigma_{*}\right)=\inf _{\sigma \in \mathcal{U}_{a d}} J(\sigma),
$$

so that $\sigma_{*}$ solves problem (4.4), i.e., $\sigma_{*}=\underline{\sigma}$.

\section{Acknowledgements}

This research was supported by the Grant IAA 100190803 of the Academy of Sciences of the Czech Republic.

\section{References}

[1] W. Allegretto, Y. Lin AND H. YANG, Finite element error estimates for nonlocal problem in American option valuation, SIAM. J. Numer. Anal., 39 (2001), pp. 834-857.

[2] L. BADEA AND J. WANG, Weak solutions for the valuation of American options, I: Uniqueness and equivalence, II: Solution existence, Analysis and Scientific Computing, Eun-Jae Park and Jongwoo Lee (Eds.), Proceedings of the 19th Daewoo Workshop in Pure Mathematics, Vol. 19, Part II, 1999, pp. 3-33.

[3] L. BADEA, On the valuation of American options, Annals of University of Craiova, Math. Comp. Sci. Ser., 31 (2004), pp. 91-97.

[4] F. BLACK AND M. SCHOLES, The pricing of options and corporate liabilities, J. Polit. Econ., 81 (1973), pp. 637-659.

[5] I. HlavÁČEK, J. Chleboun AND I. BABUŠKA, Uncertain Input Data Problems and the Worst Scenario Method, Elsevier, Amsterdam, 2004.

[6] I. HLAVÁČEK, Uncertain input data problems and the worst scenario method, Appl. Math., 52 (2007), pp. 187-196.

[7] R. C. MERTON, Theory of rational option pricing, Bell. J. Econ., 4 (1973), pp. 141-183.

[8] Q. LIN, T. LIU AND S. ZHANG, Superconvergence estimates of finite element methods for American options, Appl. Math., 54 (2009), pp. 181-202. 